\title{
ANALYSIS OF DAMAGE PROCESSES IN NANOPILLAR ARRAYS WITH HIERARCHICAL LOAD TRANSFER
}

\author{
Tomasz Derda \\ Institute of Mathematics, Czestochowa University of Technology \\ Czestochowa, Poland \\ tomasz.derda@im.pcz.pl
}

\begin{abstract}
A computational model for the damage analysis in the axially loaded nanopillar arrays was developed on the basis of the Fibre Bundle Model and hierarchical load sharing protocol. The nanopillars are characterised by random strength-thresholds drawn according to the nanoscale Weibull statistics. We study the influence of the coordination number and the number of hierarchy levels on the system strength, size of the catastrophic avalanche and probability of breakdown.
\end{abstract}

Keywords: stress transfer, probability and statistics, strength, damage mechanics, nanoscale

\section{Introduction}

The phenomena of fracture and failure of materials are ubiquitous but mostly undesirable. Especially, in materials science and engineering the fracture and damage of disordered materials are intensively investigated problems [1-3]. Under external load, materials undergo a damage process that leads to complete failure if the load increase is not stopped. In this work we study breakdown processes in axially loaded metallic nanopillar arrays $[4,5]$. The arrays of nanopillars are encountered in many areas of nanotechnology and reveal the potential applicability as components for the fabrication of electro-mechanical sense devices. To analyse failure in such arrays, we adapt the Fibre Bundle Model (FBM) which is a fundamental statistical approach for studying failure phenomena of heterogeneous materials [6-8]. The essential component of the FBM is the range and form of interaction of elements (fibres). This component is known as load transfer rule and it has considerable influence on the behaviour of the system. In most analyses, the two extreme load transfer rules are explored. The first one is the global load sharing (GLS) rule - the load carried by just destroyed element is equally transferred to all the remaining elements in the system. As opposed to this rule, for the local load sharing (LLS) rule, only nearest surviving neighbours of the failing element absorb its load. In this paper we consider clustering of the nanopillars, and therefore 
the applied load transfer rule is based on the hierarchical load sharing (HLS) [9-11]. The details of the model are presented in the following section. In the third section we present and discuss the obtained simulation results.

\section{Model of nanopillar array}

Let us consider an array consisting of $N$ longitudinal nanopillars. The system is subject to an axial mechanical loading. It is assumed that the nanopillars are of equal height and cross-sectional areas, and whose strength-thresholds $\sigma_{t h}^{i}$, $i=1,2, \ldots, N$ to an applied axial load are independent random variables. Pillarstrength-thresholds (also called critical loads) are drawn according to the nanoscale Weibull statistics $[12,13]$ :

$$
P\left(\sigma_{t h}\right)=1-\exp \left\{-n^{*}\left(\frac{\sigma_{t h}}{\sigma_{o}}\right)^{\rho}\right\}
$$

where $n^{*}$ is the number of critical defects, $\sigma_{o}$ and $\rho$ are Weibull's scale and shape parameters, respectively. It is reasonable to employ nanoscale Weibull statistics, because at the nanoscale, a small number of critical defects (possibly one critical defect) can be responsible for the damage of the specimen, independently from the specimen size [12]. By applying nanoscale Weibull statistics, simply considering $n^{*}=1$, we assume nearly defect-free structures. The Weibull modulus $\rho$, being an index of dispersion of the distribution, is 2 and $\sigma_{o}=1$.

Before the application of the external load, all the nanopillars in the array are intact. Then the system is subject to axial load $F$. In this paper two loading procedures are employed. The first one is a quasi-static loading. In this procedure the external load is uniformly increased from its initial value $(F=0)$ until the destruction of the weakest intact nanopillar under the load $\sigma_{i}$ attaining strength-threshold $\sigma_{t h}^{i}$ of this pillar. After the failure of the weakest pillar, the increase of the external load is stopped and the load from the broken pillar is transferred to other intact pillars according to a given load transfer rule. This load transfer may induce subsequent pillar failures and, as a result, next load redistributions, which can provoke further failures. If there is no failures after the load transfer, the external load has to be increased just to destroy the weakest intact pillar. The above-described procedure is repeated until all the nanopillars in the array collapse. We assume that all overloaded pillars fail simultaneously and their load is again redistributed because all failed pillars carry no load. The second employed loading procedure is the application of the finite force to the system. In this approach the external load is kept constant during the entire loading process. After the application of load $F$, all the nanopillars with strength-thresholds smaller than $F / N$ fail immediately and possible further failures are the result only of the load redistributions. 
The applied load transfer rule is based on the hierarchical load sharing protocol [11]. Suppose that the pillars are grouped in a hierarchical way (see Fig. 1). At the lowest hierarchy level, the system is seen as a set of pillars. At the second level, the pillars form groups (clusters) containing $z$ nearest neighbours from the lowest level - it's a neighbourhood of the order 1. In the third level, a group of the $z$ nearest groups from the second level is the neighbourhood of the order 2 . In the fourth level, $z$ nearest groups from the third level form neighbourhood of the order 3 etc. Therefore, we assume clustering of the pillars in the sense of their close arrangement within the groups. The effective range of interaction is restricted to pillars belonging to the same group (cluster) of the lowest possible level. This means that, if possible, the load from the broken pillar is shifted equally to all intact pillars from its neighbourhood of the order 1. If all pillars in this neighbourhood are broken, the load is equally transferred to all intact pillars from the neighbourhood of the order 2 etc. In the original version of the HLS [11], if all the elements in the cluster are broken, the load is transferred to the cluster of the higher level and is redistributed equally among all the sub-clusters of this higher-level cluster. In our calculations, the number of hierarchy levels $M$ varies from 2 to 6 . $z$ is called a coordination number. It should be noted that for ease of presentation we have illustrated the system with $z=2$ in Figure 1.

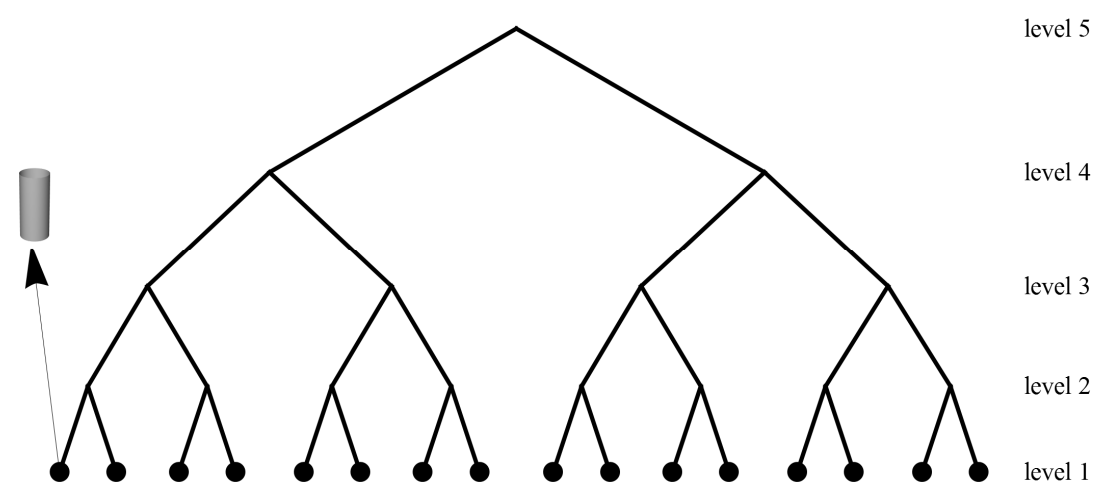

Fig. 1. Scheme of the system with coordination number $z=2$ and five levels of hierarchy. System size $N=16$

\section{Analysis of the simulation results}

Based on the FBM with HLS, a computer program was developed in order to simulate damage processes in the nanopillar arrays. Intensive computer simulations are conducted for the two loading procedures, mentioned in the previous section.

Let us consider a quasi-static loading procedure. The destruction of the system proceeds in an avalanche-like manner - each increase of the external load induces cascade or cascades of simultaneous pillar failures. The cascades of failures provoked by the increment of the external load form an avalanche $(\Delta)$. 
One of the most important properties is the strength of the system, i.e. the mean value of load at which a system composed of $N$ pillars fail. This quantity can serve as a predictor of the collapse of the system. In the quasi-static case, the system failure is caused by attaining critical load $F_{c}$. The application of the load $F_{c}$ is the last stage of the system destruction and during this stage many cascades of failures evolve to create a catastrophic (critical) avalanche $\left(\Delta_{c}\right)$.

In the following considerations, we will use values of catastrophic avalanche sizes and critical loads scaled by the initial system sizes: $\Delta_{c} / N$ and $\sigma_{c}=F_{c} / N$.

The plots of the mean critical load $\left\langle\sigma_{c}\right\rangle$ as a function of the system size $N$ are shown in Figures 2-4. As we see from these plots, mean critical load $\left\langle\sigma_{c}\right\rangle$ is a decreasing function of $N$ and numerical data can be fitted by the formula:

$$
\left\langle\sigma_{c}(N)\right\rangle=\alpha+\frac{\beta}{(\ln N)^{\delta}}
$$

where $\alpha, \beta, \delta$ are coefficients obtained from simulation results. From Figures 2 and 3 , it is also seen that the mean critical load decreases with increasing the number of hierarchy levels. This is consistent with conclusion for the original HLS that the increase in the number of hierarchy levels leads to lower strength of material [9-11]. However, it should be noted that the decrease of the critical loads slows down with the increasing number of hierarchy levels. For example, the difference between mean critical loads for $M=5$ and $M=6(z=4$, see Figure 2) is almost negligible in comparison with difference between results for $M=2$ and $M=3$.

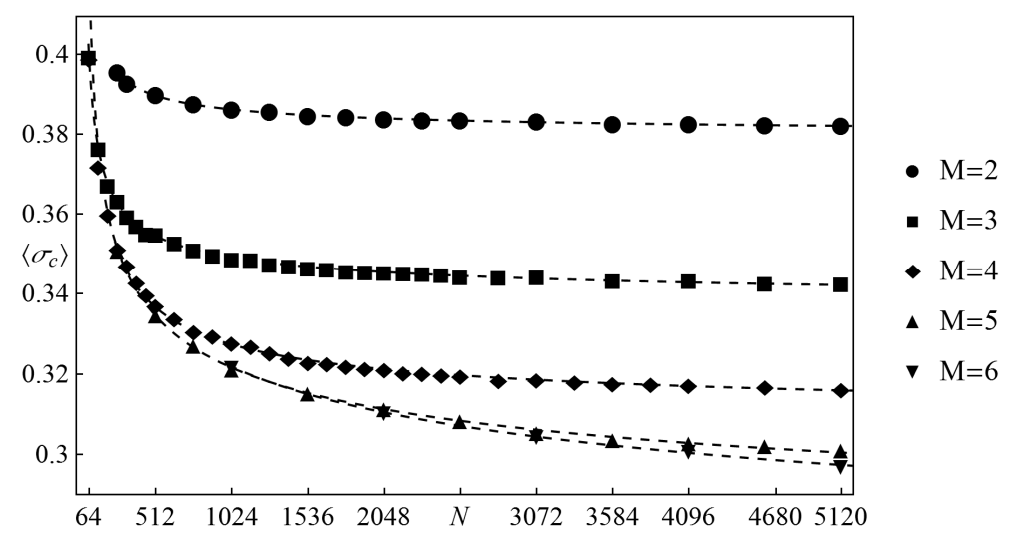

Fig. 2. The mean critical load $\left\langle\sigma_{c}\right\rangle$ versus system size $N$ for different number of hierarchy levels and $z=4$. The averages are taken from at least 1000 samples for each presented value. The dashed lines represent function (2) with parameters computed from simulations 


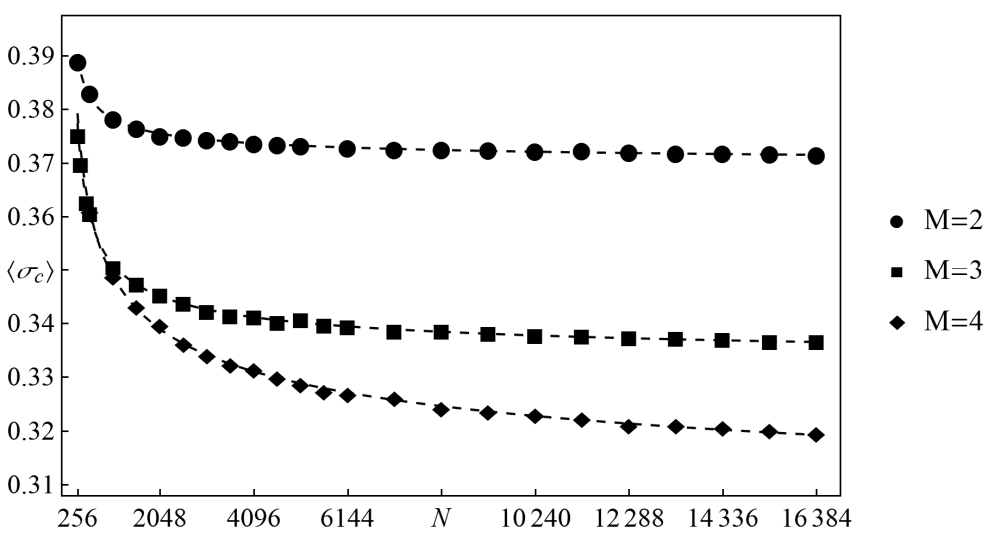

Fig. 3. The mean critical load $\left\langle\sigma_{c}\right\rangle$ versus system size $N$ for different number of hierarchy levels and $z=8$. The averages are taken from at least 1000 samples for each presented value. The dashed lines represent function (2) with parameters computed from simulations

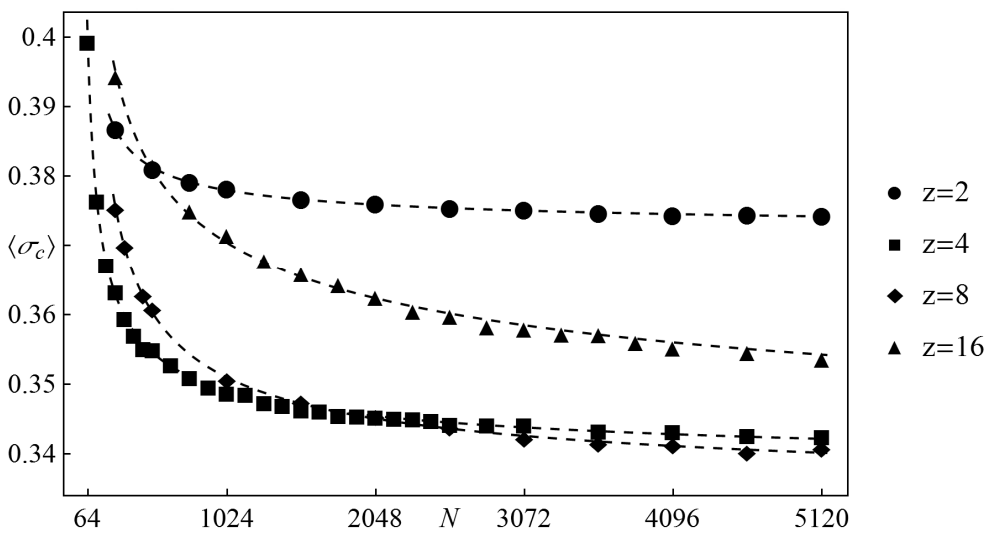

Fig. 4. The mean critical load $\left\langle\sigma_{c}\right\rangle$ versus system size $N$ for different coordination numbers and $M=3$. The averages are taken from at least 1000 samples for each presented value. The dashed lines represent function (2) with parameters computed from simulations

Here, we consider the influence of coordination number $z$ on the damage resistance of the system. Figure 4 shows mean critical loads for different values of $z$ and $M=3$. It can be seen, excluding smallest systems, that the systems with elements paired in sequential order $(z=2)$ are the strongest ones. The weakest systems are those with middle coordination numbers $(z=4$ and $z=8)$. Initially the systems with $z=4$ have the smallest $\left\langle\sigma_{c}\right\rangle$, but with increasing system size the critical loads for the arrays with $z=8$ decrease more quickly. The systems with the biggest coordination number $(z=16)$ initially are the strongest, but their $\left\langle\sigma_{c}\right\rangle$ 
decrease more rapidly than in the systems with $z=2$. Apparently, there is no ordering of critical loads in the relation to the coordination number. We will return to this in the last part of this section.
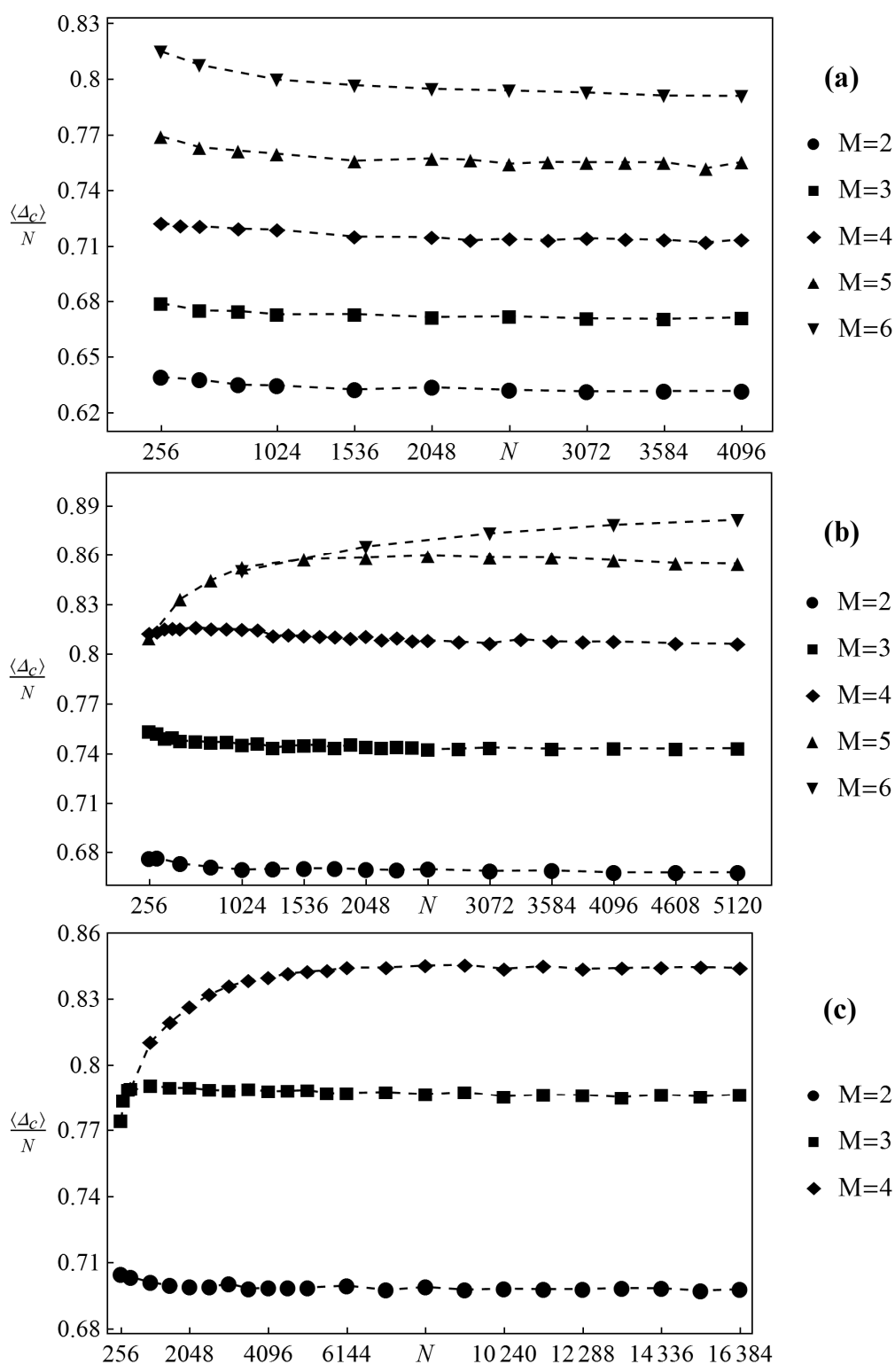

Fig. 5. The fraction of pillars destroyed during critical avalanche versus system size $N$ for $z=2$ (a), $z=4$ (b) and $z=8$ (c). The data are averaged over at least 1000 realizations for each presented value 
During a critical avalanche, an important fraction of $N$ pillars fails instantly. More specifically, most of the pillars are destroyed during the final avalanche. It is worth analysing the sizes of critical avalanches because the degree of the system destruction can be an indicator of the forthcoming system failure. Figure 5 shows the mean sizes of the normalized catastrophic avalanches as a function of $N$. One can see that the ordering reported for the critical loads is reversed the bigger the number of hierarchy levels the greater the normalized catastrophic avalanche. In some cases $\left\langle\Delta_{c}\right\rangle / N$ initially increases, but eventually it is a nonincreasing function of $N$. This conclusion is in contrast with the results obtained for both the GLS and LLS rules, for which $\left\langle\Delta_{c}\right\rangle / N$ is an increasing function of $N$ $[4,5]$. One exception to the reported rule is the system with $z=4$ and $M=6$. However, for this case, due to computational time, we did not simulate systems with $N>5120$.

In the last part of this section we analyse probabilities of breakdown of nanopillar arrays loaded by finite force $F$. For this loading procedure, the initial load $\sigma=F / N$ of all pillars is equal, and the total load $F$ is conserved during the loading process. Under such a loading procedure, three states of the system can be reached. If the initial load $\sigma=F / N$ is smaller than any of the pillar-strength-thresholds all the pillars remain intact. Application of the finite force can also lead to partial damage - the system reaches a steady state after cascades of pillar failures. Cascades may also be self-sustained until destruction of all the pillars i.e. the state of complete breakdown.

The simulation results for a different number of hierarchy levels are shown in Figures 6 and 7. It is seen from these plots that the ordering stated for the critical loads under quasi-static loading is preserved.

As shown in Figures 6-9, breakdown probability as a function of initial load per pillar $\sigma$ can be nicely fitted by the function:

$$
P(\sigma)=\frac{1}{2} \operatorname{erfc}\left(\frac{\xi-\sigma}{\sqrt{2} \omega}\right)
$$

where $\xi$ and $\omega$ are coefficients obtained from simulation results, $\operatorname{erfc}(z)$ represents complimentary error function:

$$
\operatorname{erfc}(z)=1-\operatorname{erf}(z)=\frac{2}{\sqrt{\pi}} \int_{0}^{z} \exp \left(-t^{2}\right) d t
$$

The function (3) is a cumulative distribution function of the Gaussian distribution with mean $\xi$ and variance $\omega^{2}$. 


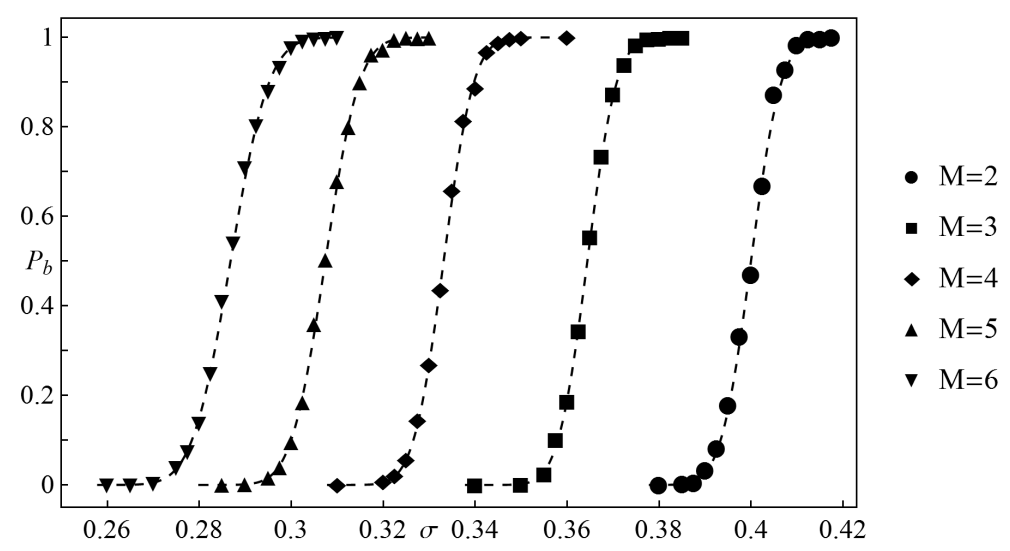

Fig. 6. Empirical breakdown probability $P_{b}$ as a function of initial load per pillar $\sigma$ for a different number of hierarchy levels and coordination number $z=2$. All presented data are calculated from 1000 statistically independent samples. System size $N=4096$.

The dashed lines represent function (3) with parameters computed from simulations

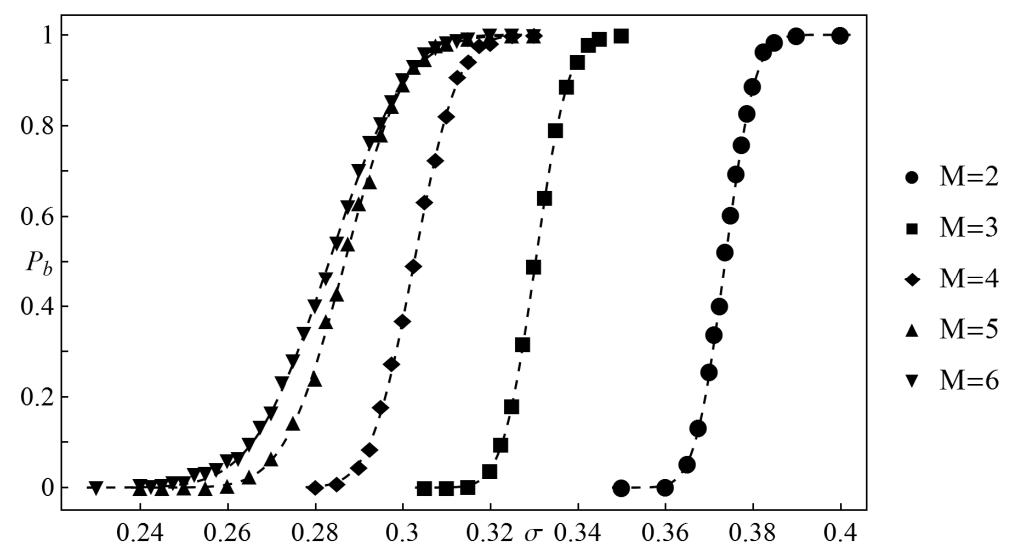

Fig. 7. Empirical breakdown probability $P_{b}$ as a function of initial load per pillar $\sigma$ for a different number of hierarchy levels and coordination number $z=4$. All presented data are calculated from 1000 statistically independent samples. System size $N=4096$.

The dashed lines represent function (3) with parameters computed from simulations

Figures 8 and 9 present probabilities of breakdown for a given number of hierarchy levels. It is seen that these probabilities are not ordered according to coordination number. This fact can be explained as follows: after destruction of the pillar the load is equally redistributed to its intact neighbours of the lowest possible level. It is appropriate to introduce a distinction between load transfer to the neighbourhood of the highest level i.e. to all the intact pillars in the system (global load transfer) and load transfer to intact neighbours of lower levels. The first load transfer is much 
more dispersed compared to the second one. It has been observed that with increase of $z$ the number of highest level load transfers decreases and the number of lower level load transfers increases. Hence, the global load transfer is partially replaced by more local transfer and this initially leads to decreasing strength. However, the local transfer becomes more and more dispersed as $z$ is increased - this, in turn, leads to increasing strength. As can be seen from Figures 4 and 8 , for $z=4$ and $z=8$, it seems that impact of the two ranges of load transfer almost compensate to give similar results.

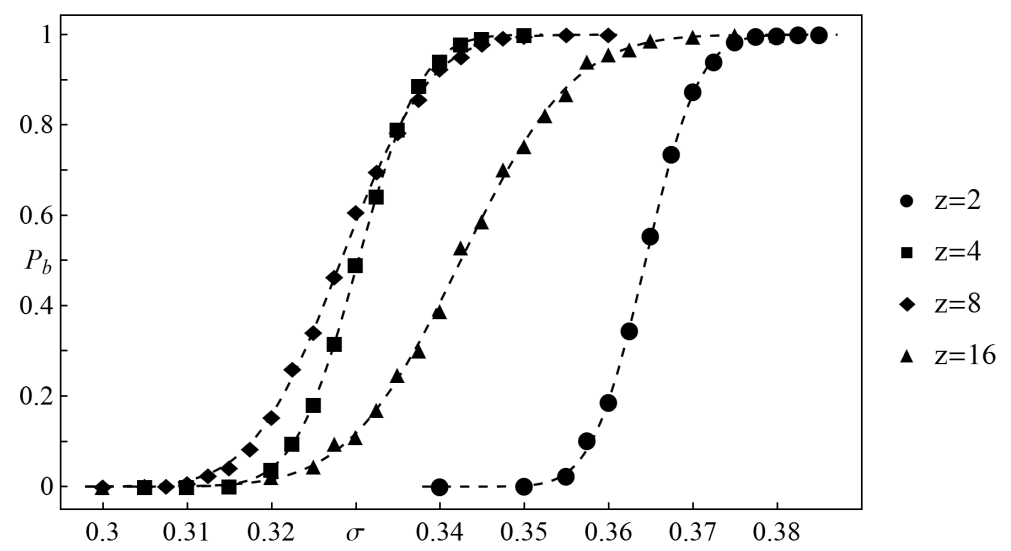

Fig. 8. Empirical breakdown probability $P_{b}$ as a function of initial load per pillar $\sigma$ for different values of $z$ and $M=3$. All presented data are calculated from 1000 statistically independent samples. System size $N=4096$. The dashed lines represent function (3) with parameters computed from simulations

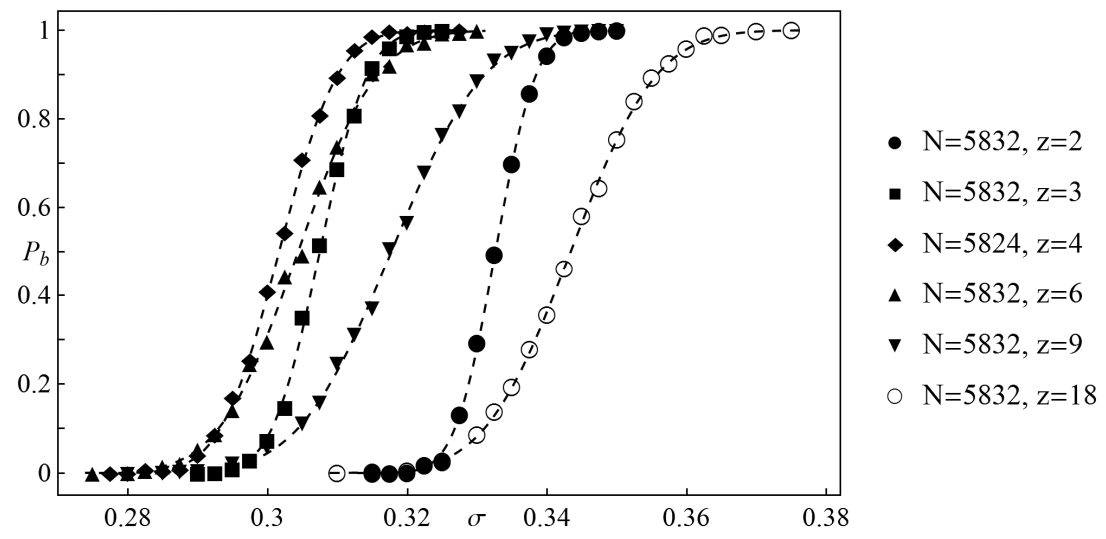

Fig. 9. Empirical breakdown probability $P_{b}$ as a function of initial load per pillar $\sigma$ for different values of $z$ and $M=4$. All presented data are calculated from 1000 statistically independent samples. The dashed lines represent function (3) with parameters computed from simulations 


\section{Conclusions}

In this paper, the computational studies of mechanical damage in the axially loaded nanopillar arrays have been discussed. Due to clustering of the pillars, we applied the load transfer rule based on the hierarchical load sharing protocol.

We have performed a series of simulations considering two different, but equivalent loading procedures. For the quasi-static loading we have noticed that the mean critical load is a decreasing function of the system size and it can be fitted by the function (2). Mean critical loads decrease as the number of hierarchy level is increased and the scaled catastrophic avalanche size is eventually nonincreasing function of $N$. For the case of finite force loading, we have shown that breakdown probabilities can be approximated by the function being a cumulative distribution function of the Gaussian distribution. We compared probabilities of breakdown for different values of the coordination number and different number of hierarchy levels.

\section{References}

[1] Herrmann H.J., Roux S. (eds.), Statistical Models for the Fracture of Disordered Media, North Holland, Amsterdam 1990 and references therein.

[2] Chakrabarti B., Benguigui L.G., Statistical Physics of Fracture and Breakdown in Disordered Systems, Claredon Press, Oxford 1997.

[3] Alava M.J., Nukala P.K.V.V., Zapperi S., Statistical models of fracture, Adv. In Physics 2006, $55,349-476$.

[4] Domański Z., Derda T., Sczygiol N., Statistics of critical avalanches in vertical nanopillar arrays, Lecture Notes in Electrical Engineering 2014, 275, 1-11.

[5] Derda T., Stochastic local load redistribution in the fibre bundle model of nanopillar arrays, J. Appl. Math. Comput. Mech. 2015, 14(4), 19-30.

[6] Pradhan S., Hansen A., Chakrabarti B.K., Failure processes in elastic fiber bundles, Rev. Mod. Phys. 2010, 82, 499-555.

[7] Hansen A., Hemmer P.C., Pradhan S., The Fiber Bundle Model: Modeling Failure in Materials, Wiley 2015.

[8] Kun F., Raischel F., Hidalgo R.C., Herrmann H.J., Extensions of fibre bundle models, Lecture Notes in Physics 2006, 705, 57-92.

[9] Newman W.I., Gabrielov A.M., Failure of hierarchical distributions of fibre bundles. I, Int. J. Fract. 1991, 50(1), 1-14.

[10] Gómez J.B., Pacheco A.F., Size-frequency distribution of earthquakes in hierarchically organized load-transfer models, Nonlin. Processes Geophys. 1997, 4, 207-211.

[11] Mishnaevsky L. Jr., Hierarchical composites: Analysis of damage evolution based on fibre bundle model, Compos. Sci. Technol. 2011,71, 450-460.

[12] Pugno N.M., Ruoff R.S., Nanoscale Weibull statistics, J. Appl. Phys. 2006, 99, 024301-4.

[13] Pugno N.M., Ruoff R.S., Nanoscale Weibull statistics for nanofibers and nanotubes, J. Aerosp. Eng. 2007, 20, 97-101. 